\title{
Application of Seismic Array Processing to Earthquake Early Warning
}

\author{
by L. Meng, R. M. Allen, and J.-P. Ampuero
}

\begin{abstract}
Earthquake early warning (EEW) systems that issue warnings prior to the arrival of strong shaking are essential in mitigating earthquake hazard. Currently operating EEW systems work on point-source assumptions and are of limited effectiveness for large events, for which ignoring finite-source effects result in magnitude underestimation. Here, we explore the concept of characterizing rupture dimensions in real time for EEW using small-aperture seismic arrays located near active faults. Back tracing array waveforms allow estimation of the extent of the rupture front (as a proxy of the rupture size) and directivity in real time, providing complementary EEW capabilities for $\mathbf{M}>7$ earthquakes to existing EEW systems. We implement it in a simulated realtime environment and analyze the 2004 M 6 Parkfield, California, earthquake recordings by the U.S. Geological Survey Parkfield dense Seismograph ARray (UPSAR) array and the 2010 M 7.2 El Mayor-Cucapah earthquake recordings by strong-motion sensors in San Diego, California. We find it important to correct for the bias in back azimuth induced by dipping structures beneath the UPSAR array, based on data from smaller events. Our estimated rupture length is $30 \%$ shorter than those inferred from other studies but still reasonable for EEW purposes. We attribute this difference to rupture directivity effects and the limited field of view of a single array. The accuracy of the approach may be improved with a network of arrays with overlapping fields of view. We demonstrate this by tracking the 2011 Tohoku earthquake rupture with two clusters of Hi-net stations in Kyushu and northern Hokkaido. The obtained results are consistent with teleseismic back-projection results and yield reasonable estimates of rupture length and directivity. Compared with other proposed finite-fault EEW approaches, the array method is less affected by the coarseness of a Global Positioning System or seismic network and provides a high-frequency characterization of the rupture that yields more suitable predictors of ground shaking for certain structures.
\end{abstract}

\section{Introduction}

Earthquake early warning (EEW) systems are a new mechanism for mitigation of seismic hazard based on issuing warnings prior to the arrival of strong ground shaking during an earthquake. All currently operating EEW systems treat earthquakes as a point source and use a magnitude-amplitude/ frequency scaling. This approach works well for moderateto-large earthquakes, but for the largest earthquakes (M > 7.5) this can lead to a significant underestimate of the ground shaking due to an underestimation of the magnitude and the absence of information about the location and extent of the fault that has ruptured. This issue was highlighted by the EEW performance during the M 9.0 Tohoku-Oki earthquake (Hoshiba et al., 2011). Using only $P$-wave amplitude information, the magnitude estimated by the Japanese EEW system saturated at $\mathbf{M}$ 8.1. Also, failing to take into account the finite-rupture propagation, the system issued warnings of strong seismic intensity only for the Tohoku region. However, the Kanto region experienced much larger ground motions than those predicted. The example of the TohokuOki earthquake demonstrates the need of characterizing the finite dimension in real time for the EEW of large earthquakes.

Among the ongoing efforts to determine the finite-fault extent in real time, Global Positioning System (GPS) approaches provide more reliable static displacements, and thus a static slip model, than seismic methods (Hudnut et al., 2002; Allen and Ziv, 2011; Colombelli et al., 2013). The FinDer approach is also proposed to determine linear fault geometry based on the difference in near/far-field seismic waveforms, provided dense station coverage (Böse et al., 2012).

Here, we explore the concept of imaging the rupture process of large earthquakes in real time using clusters of dense seismic arrays located near an active fault. The arrays can be deployed in a zigzag fashion along the fault with each array covering an effective field of view. Back tracing the waveforms of an earthquake recorded by such arrays allows the estimation of the rupture directivity, size, duration, speed, 


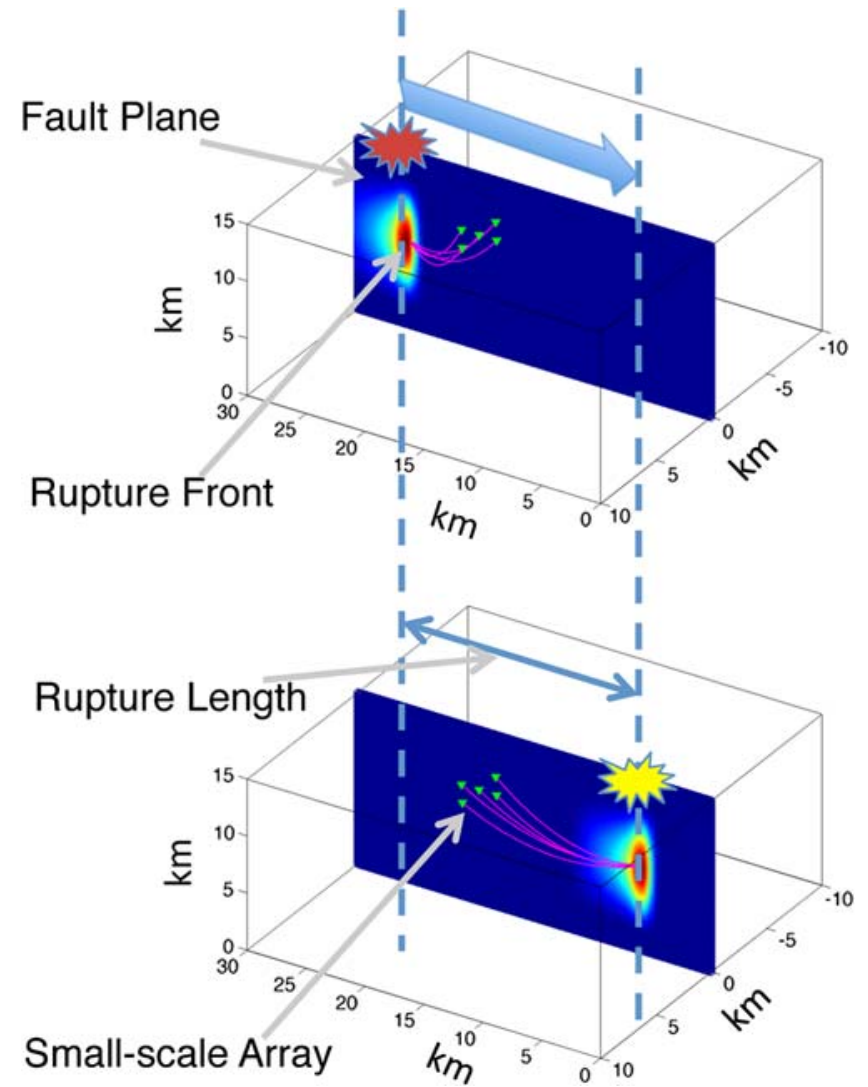

Figure 1. Concept of earthquake rupture imaging with a smallscale seismic array. The advancing slip pulse is the region of high slip velocity indicated by warm colors on the fault plane. The bang symbols represent surface projections of the rupture front. The triangles are the array stations. The curves are the ray paths of the incoming seismic waves. The dashed lines mark the spatial extent of the rupture. The color version of this figure is available only in the electronic edition.

and segmentation. The approach is now commonly used to image earthquakes recorded at teleseismic distances (Ishii et al., 2005; Meng et al., 2011) and regional distance (Vallée et al., 2008; Meng, Ampuero, Sladen, et al., 2012), with some work using it at local distances (Fletcher et al., 2006; Allmann and Shearer, 2007; Honda and Aoi, 2009). Here, we develop key aspects of the implementation of array processing at local distance in real time to estimate the rupture dimension of $\mathbf{M}>6$ earthquakes. The principle is analogous to the location and tracking of moving sources by antennas in a variety of military and civilian applications. Strong high-frequency (HF) seismic waves are typically assumed to radiate from the rupture front. Tracking the source of the HF seismic waves during large earthquakes recovers the migration of the rupture front. The trajectory of the rupture front marks the fault extent involved in the earthquake (Fig. 1). More generally, the approach provides the extent of the source area of HF radiation.

\section{Method}

The direction of arrival of waves propagating across an array can be determined by analyzing the phase of coherent seismic signals with array-processing techniques. Here, we adopt the correlation stacking technique, in which the normalized cross-correlation coefficients of all waveform pairs are beamformed (delayed and summed) instead of the waveforms themselves. This improves robustness against scattering and multipathing in the shallow crustal environment (Borcea et al., 2002; Fletcher et al., 2006). For a selected time window, the correlation coefficient $c c_{i j}$ between stations $i$ and $j$ is defined by

$$
c c_{i j}(\theta)=\frac{\sum_{t} x_{i}(t) x_{j}\left(t-\tau_{i j}(\boldsymbol{\theta})\right)}{\left(\sum_{t} x_{i}^{2} \sum_{t} x_{j}^{2}\right)^{1 / 2}},
$$

in which $x_{i}$ is the seismic signal recorded by the $i$ th station, $t$ is time index, $\tau_{i j}(\boldsymbol{\theta})$ is the time delay of the wave arrival between the station $i$ and $j$, and the sums in this equation encompass the duration of a running time window. We assume that the array aperture is much smaller than the epicentral distance and approximate the impinging wavefront as a plane. The delay as a function of the slowness vector $\boldsymbol{\theta}$ of an impinging plane wave is given by the dot product of $\tau_{i j}(\boldsymbol{\theta})=\boldsymbol{\theta} \cdot\left(r_{i}-r_{j}\right)$, in which $r_{i}$ and $r_{j}$ are the location vector of the $i$ th and $j$ th station. For fast implementation $\tau_{i j}(\boldsymbol{\theta})$ is rounded to the nearest discrete sample. We compared this approach with a more time-consuming implementation based on subsample spectral time shifting and verified that it does not introduce notable errors for signals with high sampling rate in the frequency band that we are interested in.

The correlation coefficients are then summed over all station pairs to obtain the stacked correlation coefficient, $\operatorname{scc}(\boldsymbol{\theta})$, as a function of the slowness vector of the candidate plane wave. The slowness vector contains information on both the source depth (apparent velocity) and its lateral location (back azimuth). For EEW purposes, we are primarily interested in the lateral rupture dimension and focus on the use of the back azimuth. For a given back azimuth, we define the projected lateral location as the intersection of the great circle path and a candidate fault surface (possibly nonplanar) extracted from a predefined fault catalog (e.g., the Southern California Earthquake Center community fault model). The epicenter location is provided in a real-time fashion by the conventional point-source-based EEW system (e.g., ElarmS; Kuyuk et al., 2013). If the epicenter is outside the known fault zones, the fault surface can be approximated as a linear plane with the strike determined by the interpolation of nearby faults.

The stacked correlation computed on the running windows is then analyzed as a function of the distance along the fault strike and time. At each time, the location of the peak stacked correlation is identified. If the peak values exceed a prescribed threshold (here, three times the average stacked correlation of the background noise), they are characterized as significant seismic radiators. In earthquake source imaging studies (Fletcher et al., 2006; Vallée et al., 2008; Meng, Ampuero, Stock, et al., 2012), the trajectory of the seismic radiators in the distance-time domain is used to infer the 
(a)

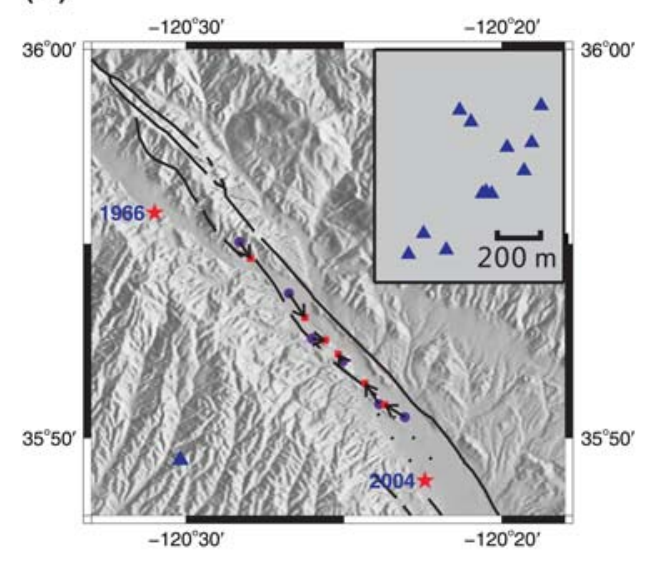

(b)

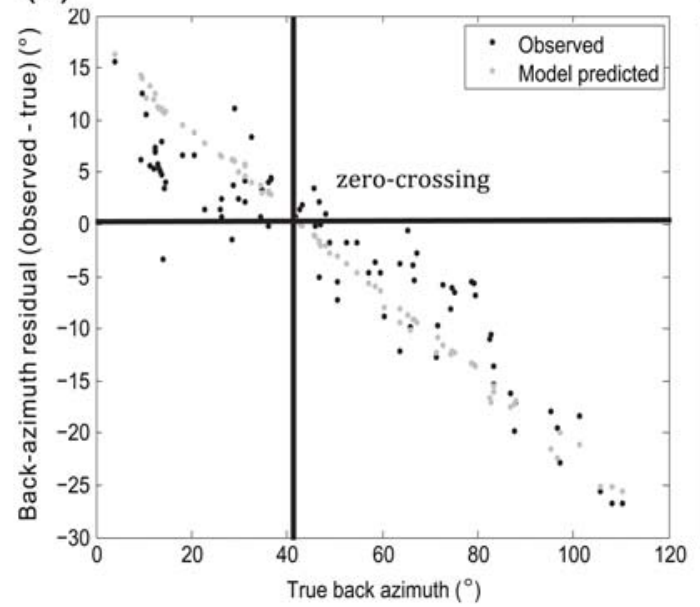

(c)

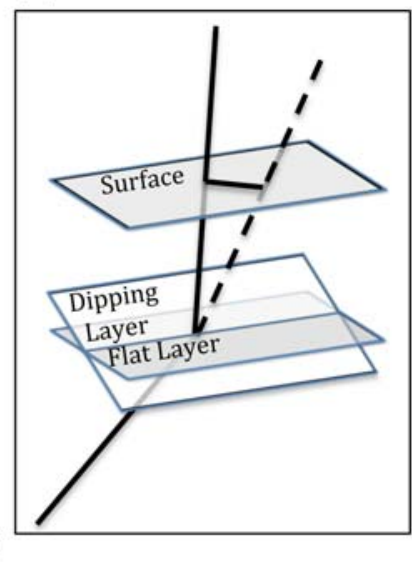

Figure 2. (a) The map view of the Parkfield area. The dots are the epicenters of five sample aftershocks of the 2004 Parkfield earthquake. The squares are the projected location of the aftershocks along a linear fault plane with azimuth retrieved by array analysis. The arrows mark direction of the bias. The stars are the epicenters of the 1966 and 2004 events. The triangle is the location of the UPSAR array with the distribution of the stations in the inset map. (b) The black dots are back-azimuth residual (observed-true) as a function of true back azimuth, and the gray dots are the anomalous azimuth predicted by a $22^{\circ}$ dipping interface toward $223^{\circ}$. (c) Sketch of ray path (dashed line) perturbed by a dipping layer and the expected ray path through the horizontal layer (solid line). The color version of this figure is available only in the electronic edition.

rupture dimension and rupture speed. However, this analysis involves human intervention to identify the rupture trend and branching patterns, which is not readily applicable in realtime implementations. We thus propose a simple algorithm to characterize the rupture dimension and directivity based on the location information. We define the rupture length as the distance between the leftmost and rightmost seismic radiators along the fault plane. The location of these two end points with respect to the hypocenter provides the rupture directivity and type (unilateral or bilateral). The information on rupture speed is not required for EEW.

\section{The 2004 M 6.0 Parkfield Earthquake}

We applied the proposed methodology to the 2004 M 6 Parkfield, California, earthquake in a simulated real-time environment. The earthquake is one of the few big events $(\mathbf{M}>6)$ that are recorded by a local small-scale seismic array (U.S. Geological Survey Parkfield dense Seismograph ARray [UPSAR]) (see Data and Resources). The array is composed of 13 stations with an aperture of about $1 \mathrm{~km}$ and spacing of roughly $200 \mathrm{~m}$ and is located at about $12 \mathrm{~km}$ from the San Andreas fault. Fletcher et al. (2006) showed the rupture kinematics can be retrieved from the UPSAR array data.

Here, before we examine the main event, we perform array analysis on the aftershocks. Figure 2 a compares the hypocenters with the locations inferred from the back azimuth retrieved by the UPSAR array. The results show the locations are biased toward the center of the rupture zone. Figure $2 \mathrm{~b}$ further shows a linear dependence of the back-azimuth residue (difference between observed and theoretical) with respect to the theoretical azimuth. Fletcher et al. (2006) propose that this bias is caused by ray-path bending due to the lateral velocity variation associated with the faultdamaged zone. However, this hypothesis requires a decreasing velocity gradient away from the fault, which is the opposite of what is observed in natural fault zones.

In the nuclear monitoring community, it has long been known that a dipping velocity structure beneath a seismic array can affect the incoming teleseismic wavefield and introduce deviations of azimuth and slowness from their expected values in a horizontally layered medium (Fig. 2b) (Niazi, 1966; Otsuka, 1966a,b). In that context, the dipping structures are typically interpreted to be the Moho discontinuity (Greenfield and Sheppard, 1969). This dipping layer effect has not been addressed in the context of array analysis at local distance. Here, we propose the azimuth bias at the UPSAR array is caused by the dipping interface between the shallow sediments and bedrock. Theory suggests a sinusoidal dependence of the backazimuth residual as a function of the true back azimuth across a $2 \pi$ range (Bondar et al., 1999; Flanagan et al., 2012). This bias pattern can be used to constrain the orientation of the dipping structure: the azimuth at which the residue crosses zero from negative to positive corresponds to the dipping direction of the interface (Lindquist et al., 2007).

Because our analysis involves only the seismicity in the Parkfield section of the San Andreas fault, the azimuth coverage is limited to about $\sim 90^{\circ}$ (Fig. 2b). Nevertheless, the back-azimuth anomaly crosses zero from positive to negative at $43^{\circ}$ (normal to the strike of the San Andreas fault), suggesting that the interface dips away $\left(223^{\circ}\right.$ from north) from the San Andreas fault. This is consistent with the topography of the UPSAR array (Fig. 2a). The dipping layer is likely caused by the fault-parallel folding deformation associated with the long-term deformation of the San Andreas fault (Mount and Suppe, 1987). The magnitude of the azimuth 

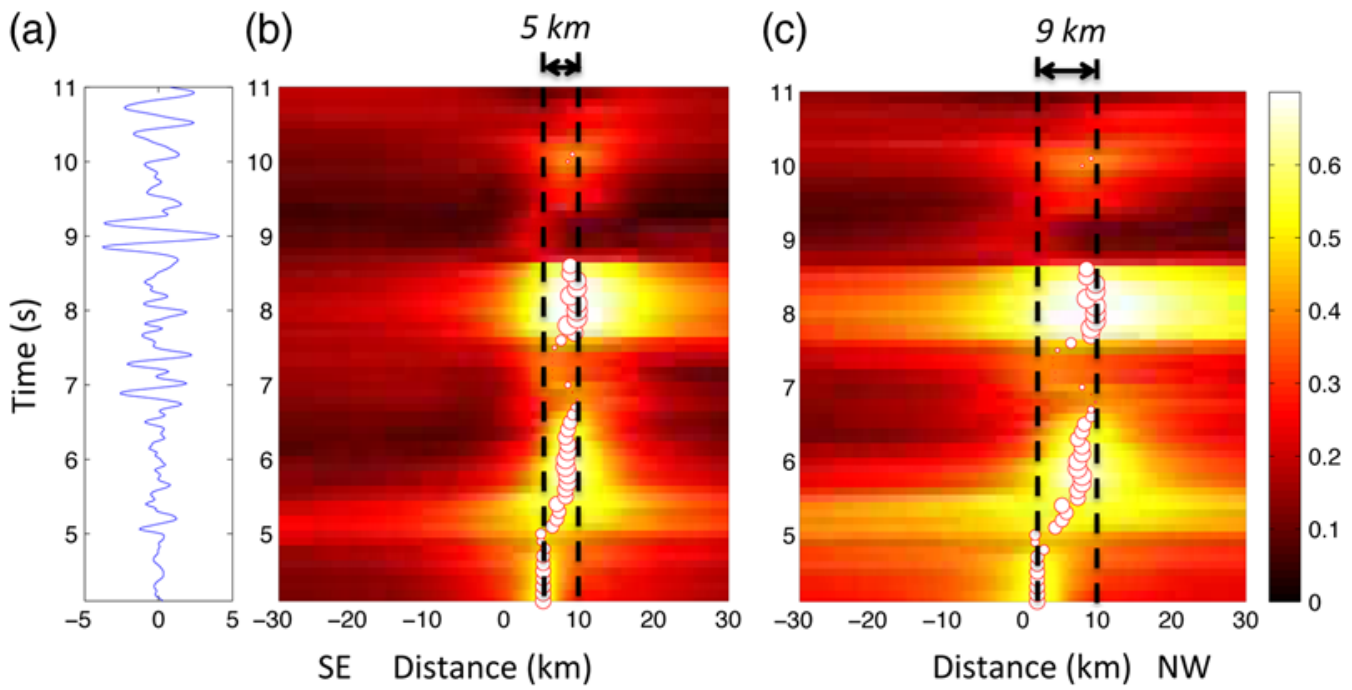

Figure 3. Stacked correlation coefficient as a function of along-strike position with respect to the hypocenter and time is shown in gray scale. (a) The seismogram at the central station of the UPSAR array. (b, c) The stacked correlation coefficient as a function of source position (projected along the fault strike, relative to the epicenter) and time (of the center of the sliding window relative to the event origin time), (a) without and (b) with correction for the dipping interface effect, respectively. The white dots are the locations of maximum correlation at each time sized by the correlation coefficient. The dashed lines mark the beginning and end of the HF energy source and give estimates of the rupture size of (b) $5 \mathrm{~km}$ and (c) $9 \mathrm{~km}$. The color version of this figure is available only in the electronic edition.

bias depends on the angle of incidence, velocity contrast across the dipping interface, and its dip angle. The incidence angles of individual aftershocks are calculated based on a 1D velocity model in the Parkfield area (Roux et al., 2005). The dip of the interface can be calculated by Snell's law given the velocity across the interface (Flanagan et al., 2012). The velocity contrast between the sediment and bedrock is too shallow to be resolved by tomography. Here we assume a ratio of 1:2 between the shear wavespeed of the shallow sediments layer and the basement rock. The inferred dip is $22^{\circ}$, consistent with that of the top layers imaged by the tomography studies (e.g., Thurber et al., 2006). This dip angle trades off with the assumed velocity contrast across the dipping interface. The true angle might be larger for a smaller shear-wavespeed ratio. Although we cannot resolve this trade-off, for the purpose of correcting the back azimuth we do not need to.

Once the dipping structure is determined, we can apply a model-based azimuth correction to the array analysis of the Parkfield mainshock, rather than the empirical correction derived by Fletcher et al. (2006) based on fitting an ad hoc smooth function to the azimuth bias. Our model-based correction enables extrapolation beyond the azimuth range represented in the empirical dataset, and because the sinusoidal shape of the calibration function is established, fewer aftershocks are required to obtain reliable corrections.

A station-specific delay correction is also applied to take into account the local $S$-wave velocity variation across the array following Fletcher et al. (2006). In practice, these corrections can be obtained by averaging the travel-time residues with respect to the best-fitting plane wave from several aftershocks. We find that this correction has a minor effect.
The resulting difference in back azimuth is much smaller than the dominant dipping layer effect.

We applied the correlation stacking to the $S$ waves recorded during the 2004 Parkfield main event in the frequency band of $0.5-8 \mathrm{~Hz}$ with a sliding window of $1 \mathrm{~s}$. The $S$ minus $P$ travel times at such close distance is shorter than the rupture duration, which prevents us from exploiting $P$ waves to track the whole rupture. The dominant frequency of the ground acceleration signals is higher than $1 \mathrm{~Hz}$. The bandwidth is narrower and the window is longer compared with Fletcher et al. (2006) to obtain a more robust image for EEW purposes. The unilateral rupture pattern is well constrained as the leftmost point is close to the hypocenter and the rightmost point is much farther to the right. Figure 3 compares the source imaging of the 2004 Parkfield earthquake with and without dipping layer correction. Ignoring the dipping structure leads to a rupture length of only $5 \mathrm{~km}$ (Fig. 3a), significantly shorter than the typical rupture length of an M 6 earthquake. Once corrected, the rupture length is $9 \mathrm{~km}$ (Fig. 3b), consistent with the estimate by Fletcher et al. (2006) based on the same dataset. Both estimates are shorter than the $13 \mathrm{~km}$ distance between the hypocenter and the northernmost subevents identified by back projection using all local stations (Allmann and Shearer, 2007). One reason for this difference is the directivity effect. The unilateral northwestward rupture propagates toward the array initially but away from the array once it passed the latitude $35^{\circ} 55^{\prime}$. This directivity effect enhances the seismic radiation of the southern rupture and weakens the northern rupture recorded at the UPSAR array. The northernmost part of the rupture thus appears too weak to be imaged by UPSAR, which results in an underestimation of the rupture length. In this case, 

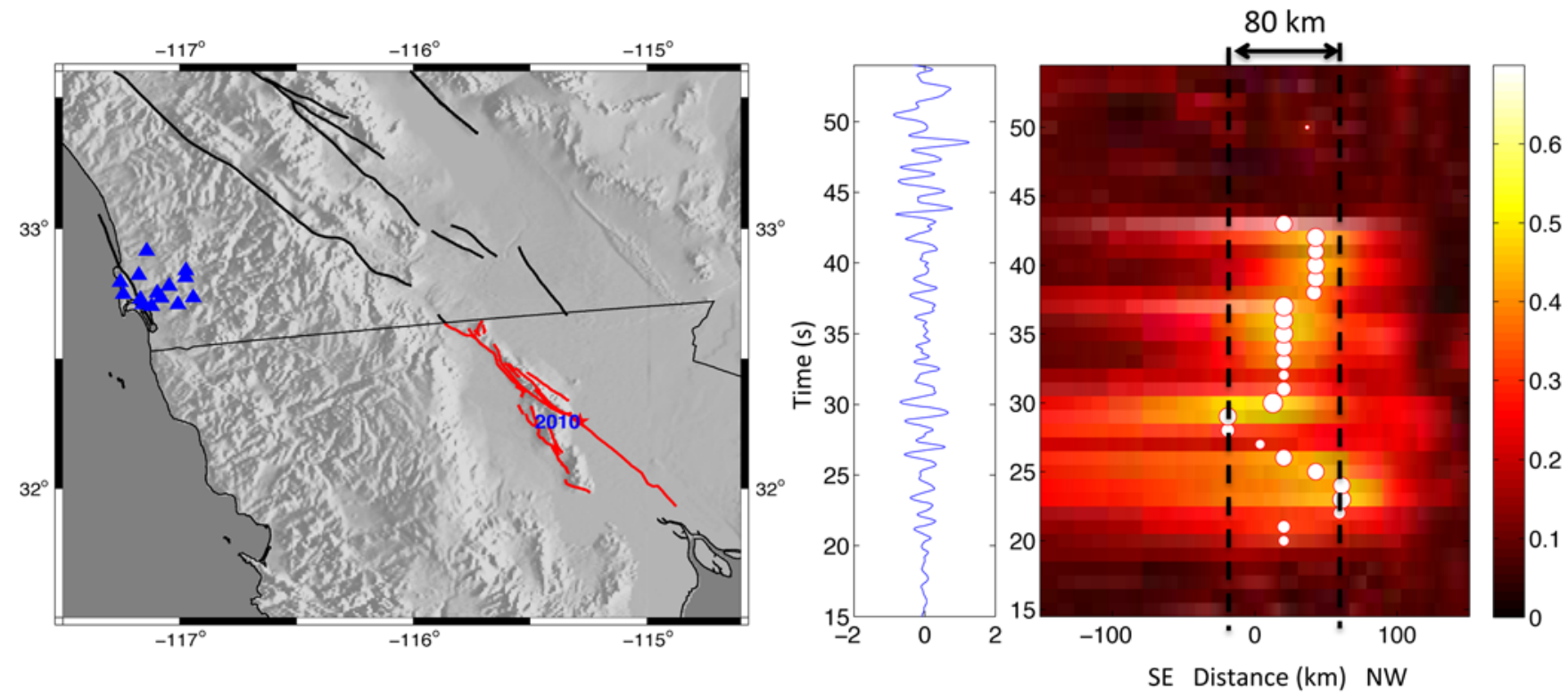

Figure 4. El Mayor-Cucapah earthquake imaged by the San Diego strong-motion array. The map shows the San Diego strong-motion array (triangles) and the fault traces of the earthquake. The panel on the right is the correlation stacking analysis of the mainshock plotted in the same fashion as Figure 3. The color version of this figure is available only in the electronic edition.

a single array is not sufficient to monitor the entire rupture area. A network of multiple small-scale arrays with partially overlapping fields of view would therefore be required to balance off the directivity effect.

\section{The 2010 M 7.2 El Mayor-Cucapah Earthquake}

As the largest recent earthquake in Baja California, the 2010 El Mayor-Cucapah earthquake is another example that can be used to demonstrate the application of array processing for EEW. It was well recorded by a group of 13 strongmotion stations in San Diego, California, with an aperture of about $30 \mathrm{~km}$ (see Data and Resources). The array is at more than $150 \mathrm{~km}$ from the rupture and outside the area of strong motion, but we can use this example as an additional test and illustration of the general approach proposed here to estimate earthquake rupture dimension. We process the $S$-wave data in the frequency range of $0.2-1 \mathrm{~Hz}$ with sliding windows of $10 \mathrm{~s}$. Because the array is not optimally oriented with respect to the fault trace, that is, it is not perpendicular to the fault, the resolution of the array is limited. The rupture was more complicated than the 2004 Parkfield event, rupturing bilaterally with a complex back and forth time history. Using our array techniques in simulated real time, a general bilateral rupture trend can be identified (Fig. 4), although the seismic radiators associated with the two rupture fronts appear intermittently. The length of the northern branch is $60 \mathrm{~km}$, consistent with finite-fault models (e.g., Wei et al., 2011). However, the southern rupture appears as only $20 \mathrm{~km}$ long, significantly shorter than that of the finite-fault models. This is again due to the directivity effect: the southeastward rupture appears weak because the rupture moves away from San
Diego. In addition, the resolution of the southern part is also lower as the great circle path of the array is even more oblique to the fault strike than the northern rupture. This example again demonstrates the value of a network of multiple arrays along the length of active faults for real-time application of this method. Overall, the $80 \mathrm{~km}$ rupture length is shorter than the $120 \mathrm{~km}$ rupture length estimated by Wei et al. (2011) but is still a reasonable estimate for EEW purpose and better than treating the event as a point source.

\section{The 2011 M 9.0 Tohoku Earthquake}

The previous two examples of application of array processing for EEW demonstrate the need for multiple arrays. Very few large earthquakes have been recorded by multiple small-scale arrays in close distance. Nevertheless, we can upscale the problem and proof the concept with a megathrust earthquake recorded by a regular seismic network. We consider the 2011 M 9.0 Tohoku earthquake, recorded by the dense Hi-net seismic network over the entire Japan.

We apply the array analysis to two clusters of Hi-net stations, one in Kyushu and one in northern Hokkaido (Fig. 5, left). The arrays are at about 1000 and $700 \mathrm{~km}$, respectively, from the hypocenter. The interstation spacing is approximately $20 \mathrm{~km}$. This setting is scaled up by roughly 100 times compared to the UPSAR/2004 Parkfield earthquake example (average spacing of $200 \mathrm{~m}$ and epicentral distance of $12 \mathrm{~km}$ ). The data are processed in the frequency band from 0.1 to $0.25 \mathrm{~Hz}$. This frequency range is less than 100 times lower than that adopted in the Parkfield example. We chose this relatively high-frequency band to compare the results with the teleseismic array back-projection results. Analysis at a 

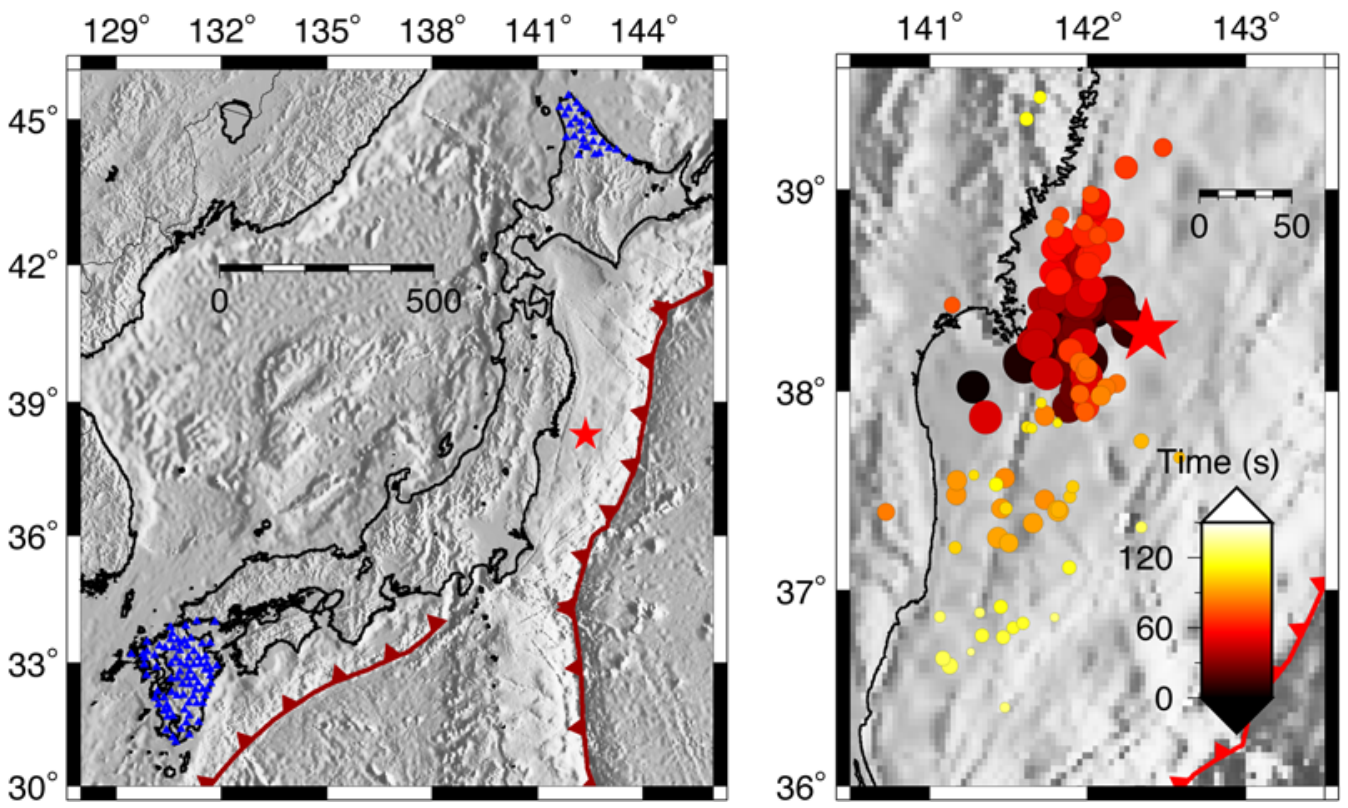

Figure 5. Tohoku earthquake imaged by clusters of Hi-net stations in Kyushu and northern Hokkaido. The map shows the selected stations (triangles) and trenches. The star denotes the hypocenter. The circles in the right panel are the locations of seismic radiation found by combining the back azimuths determined by both arrays. The brightness and size of the circles indicate the rupture time and the sum of the correlation coefficients from the two arrays, respectively. The color version of this figure is available only in the electronic edition.

lower-frequency band reproduces the large-scale features of the rupture consistently. We use the $P n$ phase, which carries the highest frequency content and proves to be well suited for earthquake source imaging at regional distance (Meng, Ampuero, Sladen, et al., 2012). Although the Tohoku rupture has been imaged by array processing with individual arrays and networks at local and regional scales (Honda et al., 2011; Nakahara et al., 2011; Maercklin et al., 2012; Roten et al., 2012), we provide the first effort to jointly image this earthquake with multiple arrays.

We performed the array analysis on recordings of the two arrays separately, and then at each epoch we intersected the directions defined by the two back azimuths to determine the location of the seismic radiation source. The significance is defined as the sum of both correlation coefficients. We apply a static correction to the back azimuths so that the initial source is located at the hypocenter. This correction is approximately $2^{\circ}$ for the Hokkaido array and $10^{\circ}$ for the Kyushu array. At this scale, this azimuth discrepancy is more likely due to lateral velocity variations rather than topography or dipping structures. The larger bias observed at the Kyushu array may reflect the lateral velocity contrast between the island arc and back-arc basin.

In general, the joint array analysis recovers the rupture process of the Tohoku earthquake reasonably well (Fig. 5, right). Both the spatial extent and rupture history are consistent with the results of high-frequency teleseismic back projection (e.g., Ishii, 2011; Koper et al., 2011; Meng et al., 2011; Yao et al., 2011; Zhang et al., 2011). The along-strike rupture length is approximately $300 \mathrm{~km}$, smaller than that of the low-frequency slip inversion (e.g., Wei et al., 2012) but consistent with the spatial extent of the source model from the high-frequency strong-motion data (Asano and Iwata, 2012). The southward rupture extent and directivity adequately explain the observed strong ground accelerations in the Kanto area. This example of the Tohoku earthquake illustrates how multiple seismic arrays enable a better characterization of the rupture length and directivity for EEW perspective.

\section{Discussion}

The array-based EEW approach can be compared with other methods characterizing the finite dimensions of earthquake rupture in real time. Based on the measurement of the static field, the GPS-based approaches (Allen and Ziv, 2011; Colombelli et al., 2013) can potentially provide valid slip estimate upon the arrival of $S$ waves. However, with limited spatial resolution and the smoothing regularization applied in the inversion, the boundary of the actual slip area can be only roughly defined. An additional approach makes use of the Zipper GPS network composed of pairs of GPS stations, one on each side of the fault, along the length of the southern San Andreas fault. It provides a direct measure of surface slip but requires GPS stations along every fault to be monitored (Hudnut et al., 2002). Similarly, by discriminating near field from far-field stations, the Finder approach (Böse et al., 2012) estimates the perimeter of the rupture zone. The boundary may again be smeared unless extremely dense station coverage is available. The array approach on the other hand provides a sharper definition of the rupture boundary achieved by exploiting the high-frequency content of the seismic wavefield. In the 2004 Parkfield example, we processed the seismograms up to $8 \mathrm{~Hz}$ (wavelength of $37 \mathrm{~m}$, assuming a shear-wave 
speed of $300 \mathrm{~m} / \mathrm{s}$ at shallow depth), implying much sharper spatial and temporal resolutions.

The view of the rupture obtained from high-frequency array analysis is not necessarily correlated to the lowfrequency aspects of the rupture process. In general, the array sees the last strong HF subevent that stands out of the coda wave of the previous HF subevents. In other words, the array sees only the strongly radiating parts of the rupture but cannot resolve a smooth rupture arrest. This partially explains why the rupture length of the Parkfield imaged by the highfrequency array studies (Fletcher et al., 2006; Allmann and Shearer, 2007; this study) is shorter than the rupture length estimated by finite-fault studies (Liu and Archuleta, 2006) and the aftershock zone (Waldhauser et al., 2004). On the other hand, if the rupture terminated abruptly, with a strong stopping phase, the high-frequency rupture size might be as large as or even larger than the low-frequency size. This is the situation of the 2010 Haiti earthquake where the highfrequency slip ruptured beyond the terminal edge of the static slip area (Meng, Ampuero, Sladen, et al., 2012). In either of these cases, the high-frequency rupture process imaged by small-aperture arrays is more directly relevant for the prediction of high-frequency ground motion. Wei et al. (2012) show that most of the ground motion of the Tohoku earthquake can be explained by only $18 \%$ of total slip located in the deep portion of the megathrust close to the Honshu Island. This deep slip is imaged by the array analysis at high frequency, and its location delineates the bottom of the long period slip region. In the example of the catastrophic Haiti earthquake, the westward high-frequency rupture is closer to Port-au-Prince, Haiti (Meng, Ampuero, Sladen, et al., 2012) than the low-frequency slip, which might have contributed to the unusually high ground motion experienced during this event (Hough et al., 2010). Thus, this HF array approach can potentially become an essential component of an EEW system, as it can constrain estimates of ground motion at the high frequencies that matter to residential areas with low story buildings (e.g., the Los Angeles area) as well as critical infrastructure and lifelines (O'Rourke et al., 2012). For this purpose, an empirical ground-motion prediction approach can be developed based on the distance and power (the observed HF energy) of the HF subevents identified by array analysis.

In terms of the practicality of a real-time implementation, the array analysis is an imaging procedure instead of an inversion, and it does not suffer from being ill posed and underdetermined. It also requires fewer assumptions about the velocity model and rupture kinematics and is therefore more suitable to be implemented in real time. In the other existing network-based finite-fault EEW approaches (GPS and Finder), information about earthquake magnitude and slip is only available following the $S$-wave arrival at network stations. In contrast, the array approach can provide a continuous update of rupture size as the earthquake unfolds, potentially saving seconds.

The calibration of the anomalous azimuth due to dipping layer beneath the array is important for the array processing- based EEW approach. The dipping structure under the UPSAR array may not be unique because fault-parallel folding is common near large strike-slip faults (Mount and Suppe, 1987). Because we propose to deploy small-scale arrays close to active faults, it is likely that these corrections are needed for all future deployments. For the purpose of the correction, a temporary high sensitivity array is needed to record the calibration events (small local events or teleseismic events) prior to the deployment of the permanent strong-motion array. For regions with low seismicity, the model-based dippinglayer correction is particularly advantageous, as it requires less calibration events than the empirical correction.

The EEW system based on the array approach still faces challenges. Particularly, a fault strike needs to be assumed based on a fault map, which is problematic if the earthquake occurs on previously unrecognized fault, although the chance of a large earthquake $(\mathbf{M}>7)$ on an unknown fault system is low. The assumption of the fault strike might be released by combining the processing of multiple arrays and triangulating the rupture location, as demonstrated here by the Tohoku earthquake example. Although site-specific back-azimuth bias are usually calibrated with aftershock data in offline processing (Honda et al., 2008), for an EEW implementation a first-order static correction can be achieved by forcing the initial back-azimuth estimates to match the hypocenter location. This procedure produces reasonable results in the Tohoku example.

The examples of the Parkfield and El Mayor-Cucapah earthquakes shown here demonstrate the incompleteness of the source imaging by a single array due to the rupture directivity effect and limited effective field of view. This results in underestimation of the rupture length. A system of multiple arrays with partially overlapping field of view is a potential solution. The joint fields of view can cover a large region and allow averaging of the rupture length estimates within the overlapping target segments to improve robustness. The bias due to the directivity effect can be mitigated with arrays located at different orientation with respect to the rupture direction.

Another challenge of all finite-fault EEW methods, including the array-based EEW approach demonstrated here, is the size of the blind zone, defined as the region that cannot receive a warning before the arrival of $S$ waves. The radius of the blind zone is predominantly dictated by the threshold of earthquake size at which a warning is issued. It takes about $10 \mathrm{~s}$ for an earthquake to grow up to $\mathbf{M} 6.5$ (with a rupture length of $30 \mathrm{~km}$, assuming a rupture speed of $3 \mathrm{~km} / \mathrm{s}$ ) and roughly $3 \mathrm{~s}$ for the $S$ wave to arrive at an array $10 \mathrm{~km}$ away from the fault (assuming an $S$ wavespeed of $3.5 \mathrm{~km} / \mathrm{s}$ ). These $13 \mathrm{~s}$ are needed to declare a warning result in a blind zone of at least $45 \mathrm{~km}$, significantly larger than that of the current California Integrated Seismic Network ShakeAlert EEW system in California (Kuyuk and Allen, 2013). We note that this blind time is dominated by rupture duration, rather than by wave travel time. Using $P$ waves instead of $S$ waves would not reduce it significantly (and is challenged by $S$ minus $P$ 
travel times shorter than rupture duration). Although the issue of the blind zone is common to all finite-fault EEW methods, these systems are not intended to provide the first warning but to send updates of rupture growth after the ShakeAlert warnings.

There is still more development necessary before arrays can be deployed for the purpose of EEW. It will be important to develop optimal designs for the array geometry and position, identify the best processing parameters (e.g., frequency band and length of sliding window), and quantify the uncertainties. Validation by synthetic tests with realistic source and path effects will help the further development of the real-time implementation strategy. Still, the initial tests shown here constitute a proof of concept for a local-array-based approach to track earthquake rupture for the purpose of EEW.

\section{Conclusion}

We developed a methodology for real-time characterization of earthquake rupture length and directivity based on seismic array data. The strategy we propose can potentially prompt more reliable EEW for large earthquakes. We implemented the proposed methodology to analyze the mainshock in a simulated real-time environment. We demonstrate the concept on the 2004 Parkfield earthquake recordings taken by the UPSAR array. We find it important to correct for the bias in back azimuth induced by dipping structures beneath the array. After calibrating the dipping-layer effect with data from smaller events, we obtained an estimated rupture length of $9 \mathrm{~km}$. This is comparable to, but a little shorter than, the distance of $13 \mathrm{~km}$ between the two main high-frequency subevents identified by back projection using all local stations. We attribute this difference in rupture length to the rupture directivity that affects the amplitude of high-frequency radiation toward UPSAR. In another example, we processed the ground accelerations of the $2010 \mathrm{El}$ Mayor-Cucapah earthquake recorded by a group of strong-motion sensors in San Diego. We find the rupture length estimate is again perturbed by the different directivity effects of the two fronts of this bilateral rupture and limited by the field of the view of a single array. Our simulated real-time estimate of $80 \mathrm{~km}$, a little less than the $120 \mathrm{~km}$ estimate from other studies, is still reasonable for EEW purposes. A network of arrays with overlapping fields of view can potentially improve the robustness and accuracy, which we demonstrated by jointly imaging the 2011 Tohoku earthquake with two clusters of Hi-net stations in Kyushu and Hokkaido: the recovered rupture process is consistent with teleseismic back-projection imaging results, and the inferred rupture length of $300 \mathrm{~km}$ and southward directivity are satisfactory for EEW purposes. We compared the benefits of the array-based EEW approach with other realtime finite-fault EEW solutions based on GPS and seismic data. The array method constrains high-frequency aspects of the rupture that are complementary to the current EEW system and a more suitable ground-motion predictor in certain cases.

\section{Data and Resources}

The Center for Engineering Strong Motion Data (CESMD) is accessed to download the strong-motion data of the El Mayor-Cucapah earthquake (http://strongmotioncenter.org/; last accessed April 2013). The seismograms of the Parkfield earthquake recorded at the UPSAR array is available at the U.S. Geological Survey national strong-motion project (http://nsmp.wr.usgs.gov/; last accessed February 2013). The broadband seismograms of the Tohoku earthquake is acquired from the Japanese Hi-net (http://www.hinet.bosai.go. jp; last accessed February 2014).

\section{Acknowledgments}

This work was funded by the Gordon and Betty Moore Foundation through Grant GBMF3024 to University of California Berkeley. We thank Nathan Simmons for valuable discussions about the dipping layer effect. We thank Paul Spudich and Joe Fletcher for providing the back-azimuth estimates of the 2004 Parkfield earthquake and its aftershocks.

\section{References}

Allen, R. M., and A. Ziv (2011). Application of real-time GPS to earthquake early warning, Geophys. Res. Lett. 38, no. 16, doi: 10.1029/ 2011 GL047947.

Allmann, B. P., and P. M. Shearer (2007). A high-frequency secondary event during the 2004 Parkfield earthquake, Science 318, no. 5854, 1279-1283, doi: 10.1126/science.1146537.

Asano, K., and T. Iwata (2012). Source model for strong ground motion generation in the frequency range $0.1-10 \mathrm{~Hz}$ during the 2011 Tohoku earthquake, Earth Planets Space 64, no. 12, 1111-1123.

Bondar, I., R. North, and G. Beall (1999). Teleseismic slowness-azimuth station corrections for the international monitoring system seismic network, Bull. Seismol. Soc. Am. 89, no. 4, 989-1003.

Borcea, L., G. Papanicolaou, C. Tsogka, and J. Berryman (2002). Imaging and time reversal in random media, Inverse Probl. 18, no. 5, 1247-1279.

Böse, M., T. H. Heaton, and E. Hauksson (2012). Real-time finite fault rupture detector (FinDer) for large earthquakes, Geophys. J. Int. 191, no. 2, 803-812, doi: 10.1111/j.1365-246X.2012.05657.x.

Colombelli, S., R. M. Allen, and A. Zollo (2013). Application of real-time GPS to earthquake early warning in subduction and strike-slip environments, J. Geophys. Res. 118, doi: 10.1002/jgrb.50242.

Flanagan, M., S. Myers, and N. Simmons (2012). Model-based corrections to observed back azimuth and slowness observations from a dipping Mohorovicic discontinuity, in Monitoring Research Review, Albuquerque, New Mexico, 18 September-20 September 2012, LLNL-CONF-563592.

Fletcher, J. B., P. Spudich, and L. M. Baker (2006). Rupture propagation of the 2004 Parkfield, California, earthquake from observations at the UPSAR, Bull. Seismol. Soc. Am. 96, no. 4B, S129-S142, doi: 10.1785/ 0120050812 .

Greenfield, R., and M. Sheppard (1969). The Moho depth variations under the LASA and their effect on dT/d $\Delta$ measurements, Bull. Seismol. Soc. Am. 59, no. 1, 409-420.

Honda, R., and S. Aoi (2009). Array back-projection imaging of the 2007 Niigataken Chuetsu-oki earthquake striking the world's largest nuclear power plant, Bull. Seismol. Soc. Am. 99, no. 1, 141-147, doi: 10.1785/ 0120080062.

Honda, R., S. Aoi, H. Sekiguchi, and H. Fujiwara (2008). Imaging an asperity of the 2003 Tokachi-oki earthquake using a dense strong-motion seismograph network, Geophys. J. Int. 172, no. 3, 1104-1116, doi: 10.1111/j.1365-246X.2007.03702.x.

Honda, R., Y. Yukutake, H. Ito, M. Harada, T. Aketagawa, and A. Yoshida (2011). A complex rupture image of the 2011 off the Pacific coast of 
Tohoku earthquake revealed by the MeSO-net, Earth Planets Space 63, no. 7, 583-588, doi: 10.5047/eps.2011.05.034.

Hoshiba, M., K. Iwakiri, N. Hayashimoto, and T. Shimoyama (2011). Outline of the 2011 off the Pacific coast of Tohoku earthquake $\left(M_{\mathrm{w}} 9.0\right)$ Earthquake early warning and observed seismic intensity, Earth Planets Space 63, no. 7, 547-551, doi: 10.5047/eps.2011.05.031.

Hough, S. E., J. R. Altidor, D. Anglade, D. Given, M. G. Janvier, J. Z. Maharrey, M. Meremonte, B. S.-L. Mildor, C. Prepetit, and A. Yong (2010). Localized damage caused by topographic amplification during the 2010 M 7.0 Haiti earthquake, Nat. Geosci. 3, no. 11, 778-782, doi: 10.1038/ngeo988.

Hudnut, K. W., G. J. Anderson, A. Aspiotes, N. E. King, R. A. Moffitt, and K. F. Stark (2002). GPS fault slip sensors, Asia-Pacific Econ. Coop. Symp. Conffronting Urban Earthquakes - Seism. Early Warn., Taipei, Taiwan, 28-29 November.

Ishii, M. (2011). High-frequency rupture properties of the $M_{\mathrm{w}} 9.0$ off the Pacific coast of Tohoku earthquake, Earth Planets Space 63, no. 7, 609-614, doi: 10.5047/eps.2011.07.009.

Ishii, M., P. M. Shearer, H. Houston, and J. E. Vidale (2005). Extent, duration and speed of the 2004 Sumatra-Andaman earthquake imaged by the Hi-Net array, Nature 435, no. 7044, 933-936, doi: 10.1038/ nature 03675 .

Koper, K. D., A. R. Hutko, T. Lay, C. J. Ammon, and H. Kanamori (2011). Frequency-dependent rupture process of the $2011 M_{\mathrm{w}} 9.0$ Tohoku earthquake: Comparison of short-period $P$ wave backprojection images and broadband seismic rupture models, Earth Planets Space 63, no. 7, 599-602, doi: 10.5047/eps.2011.05.026.

Kuyuk, H. S., and R. M. Allen (2013). Optimal seismic network density for earthquake early warning: A case study from California, Seismol. Res. Lett. 84, no. 6, 946-954, doi: 10.1785/0220130043.

Kuyuk, S., R. M. Allen, H. Brown, M. Hellweg, I. Henson, and D. Neuhauser (2013). Designing a network-based earthquake early warning system algorithm for California: ElarmS-2, Bull. Seismol. Soc. Am. 104, 162-173, doi: 10.1785/0120130146.

Lindquist, K. G., I. M. Tibuleac, and R. A. Hansen (2007). A semiautomatic calibration method applied to a small-aperture Alaskan seismic array, Bull. Seismol. Soc. Am. 97, no. 1B, 100-113, doi: 10.1785/ 0120040119.

Liu, P., and R. Archuleta (2006). Kinematic inversion of the 2004 M 6.0 Parkfield earthquake including an approximation to site effects, Bull. Seismol. Soc. Am. 96, no. 4B, S143-S158, doi: 10.1785/0120050826.

Maercklin, N., G. Festa, S. Colombelli, and A. Zollo (2012). Twin ruptures grew to build up the giant 2011 Tohoku, Japan, earthquake, Sci. Rep. 2, 709, doi: 10.1038/srep00709.

Meng, L., J.-P. Ampuero, A. Sladen, and H. Rendon (2012). High-resolution backprojection at regional distance: Application to the Haiti M 7.0 earthquake and comparisons with finite source studies, J. Geophys. Res. 117, no. B4, B04313, doi: 10.1029/2011JB008702.

Meng, L., J.-P. Ampuero, J. Stock, Z. Duputel, Y. Luo, and V. C. Tsai (2012). Earthquake in a maze: Compressional rupture branching during the $2012 M_{\mathrm{w}}$ 8.6 Sumatra earthquake, Science 337, no. 6095, 724-726, doi: $10.1126 /$ science. 1224030 .

Meng, L., A. Inbal, and J.-P. Ampuero (2011). A window into the complexity of the dynamic rupture of the $2011 M_{\mathrm{w}} 9$ Tohoku-Oki earthquake, Geophys. Res. Lett. 38, no. 7, L00G07, doi: 10.1029/2011GL048118.

Mount, V. S., and J. Suppe (1987). State of stress near the San Andreas fault Implications for wrench tectonics, Geology 15, no. 12, 1143-1146, doi: 10.1130/0091-7613(1987)15<1143:SOSNTS >2.0.CO;2.

Nakahara, H., H. Sato, T. Nishimura, and H. Fujiwara (2011). Direct observation of rupture propagation during the 2011 off the Pacific coast of Tohoku earthquake $\left(M_{\mathrm{w}}\right.$ 9.0) using a small seismic array, Earth Planets Space 63, no. 7, 589-594, doi: 10.5047/eps.2011.06.002.

Niazi, M. (1966). Corrections to apparent azimuths and travel-time residuals for a dipping Mohorovicic discontinuity, Bull. Seismol. Soc. Am. 56, no. $2,491-509$.

O'Rourke, T., S.-S. Jeon, S. Toprak, M. Cubrinovski, and J. K. Jung (2012). Underground lifeline system performance during the Canterbury earth- quake sequence, in 15th World Conference on Earthquake Engineering, Lisbon, Portugal, Lisbon, 24-28 September 2012.

Otsuka, M. (1966a). Azimuth and slowness anomalies of seismic waves measured on the Central California Seismographic Array, part I: Observations, Bull. Seismol. Soc. Am. 56, no. 1, 223-239.

Otsuka, M. (1966b). Azimuth and slowness anomalies of seismic waves measured on the Central California seismographic array, Part II: Interpretation, Bull. Seismol. Soc. Am. 56, no. 3, 655-675.

Roten, D., H. Miyake, and K. Koketsu (2012). A Rayleigh wave back-projection method applied to the 2011 Tohoku earthquake, Geophys. Res. Lett. 39, no. 2, doi: 10.1029/2011GL050183.

Roux, P., K. Sabra, P. Gerstoft, and W. A. Kuperman (2005). P-waves from cross-correlation of seismic noise, Geophys. Res. Lett. 32, no. 19, L19303, doi: 10.1029/2005GL023803.

Thurber, C., H. Zheng, F. Waldhuser, J. Hardebeck, A. Michael, and D. Eberhart-Philips (2006). Three-dimensional compressional wavespeed model, earthquake relocations, and focal mechanisms for the Parkfield, California, region, Bull. Seismol. Soc. Am. 96, no. 4B, S38-S49, doi: $10.1785 / 0120050825$.

Vallée, M., M. Landès, N. M. Shapiro, and Y. Klinger (2008). The 14 November 2001 Kokoxili (Tibet) earthquake: High-frequency seismic radiation originating from the transitions between sub-Rayleigh and supershear rupture velocity regimes, J. Geophys. Res. 113, no. B7, B07305, doi: 10.1029/2007JB005520.

Waldhauser, F., W. Ellsworth, D. P. Schaff, and A. Cole (2004). Streaks, multiplets, and holes: High-resolution spatio-temporal behavior of Parkfield seismicity, Geophys. Res. Lett. 31, no. 18, L18608, doi: 10.1029/2004GL020649.

Wei, S., E. Fielding, S. Leprince, A. Sladen, J. P. Avouac, D. Helmberger, E. Hauksson, R. Chu, M. Simons, K. Hudnut, T. Herring, and R. Briggs (2011). Superficial simplicity of the 2010 El Mayor-Cucapah earthquake of Baja California in Mexico, Nat. Geosci. 4, no. 9, 615618, doi: 10.1038/ngeo1213.

Wei, S., R. Graves, D. Helmberger, J.-P. Avouac, and J. Jiang (2012). Sources of shaking and flooding during the Tohoku-Oki earthquake: A mixture of rupture styles, Earth Planet. Sci. Lett. 333/334, 91-100, doi: 10.1016/j.eps1.2012.04.006.

Yao, H., P. Gerstoft, P. M. Shearer, and C. Mecklenbräuker (2011). Compressive sensing of the Tohoku-Oki $M_{\mathrm{w}} 9.0$ earthquake: Frequency-dependent rupture modes, Geophys. Res. Lett. 38, no. 20, doi: 10.1029/2011GL049223.

Zhang, H., Z. Ge, and L. Ding (2011). Three sub-events composing the 2011 off the Pacific coast of Tohoku earthquake ( $\left.M_{\mathrm{w}} 9.0\right)$ inferred from rupture imaging by back-projecting teleseismic $P$ waves, Earth Planets Space 63, no. 7, 595-598, doi: 10.5047/eps.2011.06.021.

Department of Earth, Planetary, and Space Sciences

University of California, Los Angeles

Los Angeles, California 90095

meng@ess.ucla.edu

(L.M.)

Berkeley Seismological Laboratory

University of California, Berkeley

Berkeley, California 94703

(R.M.A.)

Seismological Laboratory

California Institute of Technology

Pasadena, California 91125

(J.-P.A.)

Manuscript received 25 October 2013; Published Online 16 September 2014 